\title{
Single-pump multiwavelength hybrid Raman-EDF laser using a non-adiabatic microfiber interferometer
}

\author{
M.I. Md Ali ${ }^{1}$, Nani Fadzlina Naim ${ }^{2}$, Muhammad Faiz Ibrahim ${ }^{3}$ \\ 1,2,3 Faculty of Electrical Engineering, Universiti Teknologi MARA, Malaysia
}

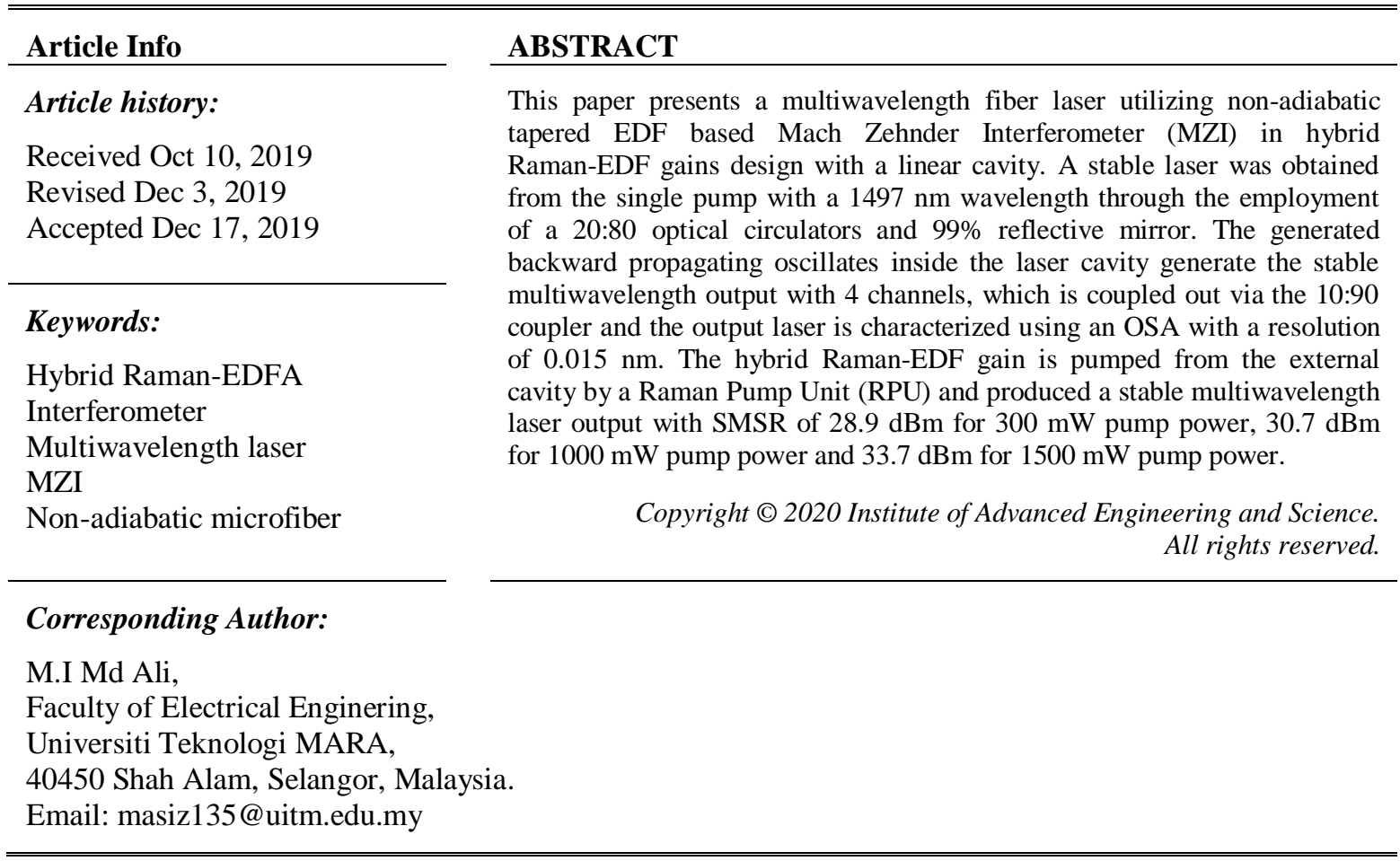

\section{INTRODUCTION}

Optical fiber technology was designed not only for optical communication, but other applications including sensor system [1], navigation system [2], manufacturing [3] and many more. The role of optical fibers in other fields has stirred research in the photonics and laser technology. Before the invention of laser, the light emitting diode (LED) is used as the transmitter, but due to the increase of the data capacity, LED is no longer an ideal transmitter since its broad spectrum limits the ability to increase the transmission capacity [4]. Therefore, the laser is an ideal solution due to its narrow linewidth, high output power, compatibility to optical to electrical conversion and availability in various frequencies [5]. Laser can be found in many forms such as semiconductor laser, gas laser and optical fiber laser [6, 7]. Among all, optical fiber laser has numerous advantages including ultra-narrow emission spectrum and high output power that is particularly useful especially for high accuracy technologies [5].

As part of optical fiber laser, multiwavelength fiber laser also generates interest, especially towards improving the wavelength division multiplexing (WDM) system. These lasers also have a large potential in the fiber-optic test and measurement of WDM components. There is work reported on fiber laser using the effect of Stimulated Brillouin Scattering [8]. However, Brillouin effects requires high pump power to achieve threshold conditions for lasing. The fundamentals for such multiwavelength optical sources are: a high number of channels over the large wavelength span, moderate output powers per channel with a good optical signal to interference ratio (OSNR) and spectral flatness, single longitudinal mode operation of each laser line and accurate placement on the frequency grid [9, 10]. Accomplishing all these requirements simultaneously is a difficult task, and many different approaches using semiconductor or Erbium-Doped Fiber (EDF) technology has been proposed and tried out in order to obtain multiwavelength laser oscillation [11]. 
Nevertheless, an intrinsic disadvantage of EDF is it possesses a strong homogenous line broadening and cross-saturation gain at room temperature $[12,13]$. In this experiment, a multi-wavelength fiber laser based on a hybrid gain medium of EDF and Raman has been introduced to suppress the homogenous line broadening of Erbium ions and able to operate in several wavelength regions; $\mathrm{C}$ band and $\mathrm{L}$. This region has attracted the most attention because it coincides with the low loss region of silica fibers used for optical communications.

To produce a multiwavelength laser output, an interferometer is required within the laser cavity. An interferometer is a technique that employs the superposition of electromagnetic waves [14]. There are a number of categories in utilizing this technique, which differ in terms of the properties that includes the properties of EM waves, the propagation path and also the method of splitting and combining the waves [14]. One of the methods of interferometer technique if the splitting involving only the amplitude waves, it called as amplitude splitting; such as Mach Zehnder interferometer (MZI), Fabry Perot and Michelson interferometer $[15,16]$. The technique is different with the splitting of wave front such as Young slit experiment and also Lloyd mirror. Generating multiwavelength fiber laser utilizing both techniques has been demonstrated through various experiments $[15,16]$.

The major drawbacks of these methods are the high losses introduced by integrating the interferometer inside the cavity. Besides, the physical construction of the techniques is challenging. Hence, this work introduced tapered EDF based on Mach Zehnder interferometer. In this work, the fiber acts as an MZI as well as amplification element in the cavity as a section of EDF is tapered. The tapered EDF based MZI can be physically understood as an interferometer. The selection of this technique is based on its advantages including; easy to fabricate, compactness, low cost, low loss and possibility for future implementation.

\section{RESEARCH METHOD}

A schematic diagram of the multiwavelength fiber laser is shown in Figure 1. The operation of multiwavelength laser started when 1497nm Raman Pump Unit (RPU) with $0.2 \mathrm{~W}$ - $1.8 \mathrm{~W}$ pump power respectively get associated with $10 \%, 20,30 \%, 40 \%, 50 \%, 60 \%, 70 \%, 80 \%$ and $90 \%$ coupler to produce a laser spectrum in the Raman-EDF gains medium. The variety of coupler allow the pump wavelength to enter the fiber transmission system without disturbing the signal when Raman pump spliced into the laser transmission system. The RPU continually pumps the fiber and as pulses pass through the system, they are amplified through the hybrid Raman-EDF medium while the pump light is depleted.

RPU are based on stimulated Raman scattering, which occurs in fibers at high powers. When pumping is direct to the upper laser state, the lowest lying level experience rapid relaxation from which laser action is produced [17]. In this case, the laser output occur in the region of $1510 \mathrm{~nm}-1630 \mathrm{~nm}$ consequently. The higher levels of that state are not significantly populated and hence can serve as a lower level of population inversion. The population then rapidly decays to the lower level at that state [18].

As illustrated in the Figure 1, a section of SMF with a distance of $11 \mathrm{~km}$ and $17 \mathrm{~m}$ EDF is used to form a hybrid Raman-EDF cavity to produce a stable laser output. For a signal wavelength at $1597 \mathrm{~nm}$, the pump wavelength should be about $1497 \mathrm{~nm}$ to ensure the highest gain. To achieve a gain flatness over a broad range of signal frequencies, multiple pumping mechanism are usually used in practical systems. For example, the variety number of coupler will produce a forward and backward pumping mechanism with the result of different type of gain medium produced [19].

To achieve this laser it is necessary to contain photons within the laser medium and maintain the conditions for coherence [20]. This is accomplished by placing mirrors and circulator at either end of the amplifying medium, as illustrated in Figure 1. The optical cavity formed is more analogous to an oscillator than an amplifier as it provides positive feedback of the photons by reflection at the mirrors and circulator at the end of the cavity. Hence the optical signal is fed back many times while receiving amplification as it passes through the Hybrid Raman-EDF gain medium. Although the amplification of the signal from a single pass through the medium is quite small, after multiple passes the net gain can be large. A stable output is obtained at saturation when the optical gain is exactly matched by the losses incurred in the amplifying medium. In addition, the performance and of the laser will be discussed further in this paper in term of stability, output power, SMSR, lasing threshold, spectral spacing and line width.

In this experiment, single-mode fibers were tapered to a diameter of a $10 \mu \mathrm{m}$ (waist diameter) causing multiple cladding modes to be stimulated and to propagate through the taper waist (interference). The presence of multiple modes creates an interference pattern in the output signal. As presented in Figure 2, tapered regions serve as interference interface, [21]. The taper fiber interference spectrum analyzed in the work is obtained by the interaction between two ways the fundamental mode HE11 ( $\left.\mathrm{I}_{1}\right)$ and a HOM (High Order Mode) HEım ( $\left.I_{2}\right)$. This modal interference is shown by the equation: 


$$
I=I 1+I_{2}+2 \sqrt{I 1 * I 2} \cos (\phi)
$$

Here the phase $(\Phi)$ is the difference between the propagation constant of the modes involves and can be expressed in terms of the effective refractive index difference of both modes:

$$
\phi=\frac{2 \pi \Delta n L}{\lambda}
$$

Where the difference between the effective refractive index of fundamental mode $\left(\mathrm{n}_{1}\right)$ and the HOM $\left(\mathrm{n}_{2}\right)$ is expressed by $\triangle n=\mathrm{n}_{2}-\mathrm{n}_{1}, \lambda$ is wavelength operation and $\mathrm{L}$ is the length of the fiber waist for the tapered fiber. The phase difference between the modes is generated when the tapered down area generated these modes and they recombine to a single mode again after the tapered-up zone.

The multi-reflection process that happen in the tapered based EDF is unique and it produces a transfer function much different from the other interferometer such as Fabry-Pérot [22, 23], Michelson and Sagnac configurations $[24,25]$. The narrow core along the fiber phase is extremely high sensitivity and relatively low loss and for this reason, the tapered based EDF filter has a greater sensitivity and one of the best results to produce multiwavelength laser compared to the other techniques.

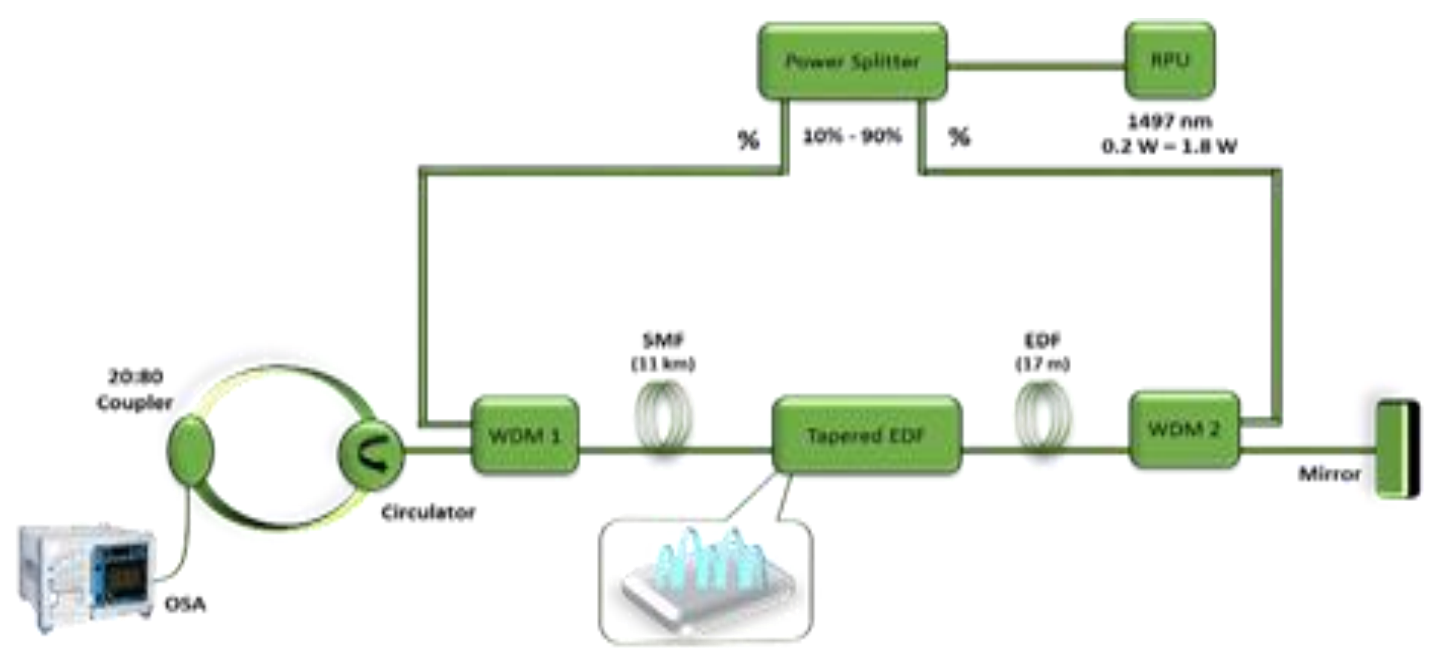

Figure 1. Schematic diagram of multiwavelength fiber laser

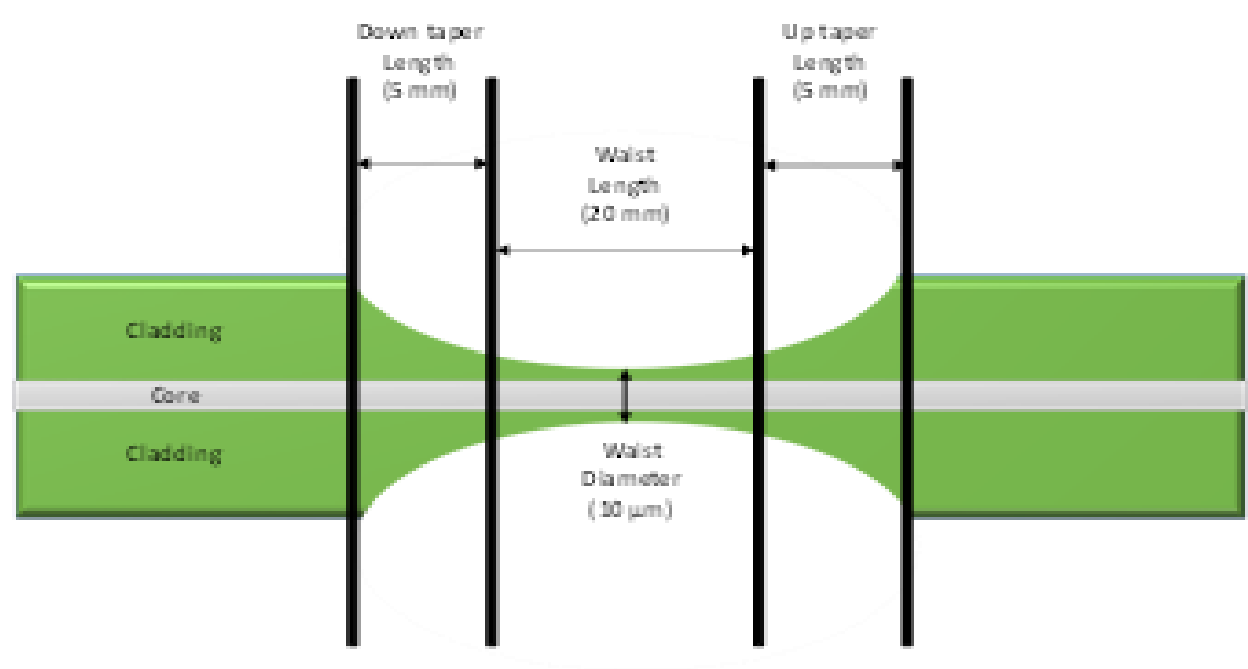

Figure 2. Tapered Single mode fiber as Mach-Zehnder Interferometer 


\section{RESULTS AND ANALYSIS}

The transmission spectrum of the tapered fiber using the amplified spontaneous emission (ASE) source was shown in Figure 3 and the result shows the power variations are largely sinusoidal in the data. In order to determine the best result on the transmission spectrum, the taper was fixed in a flow cell trough and held in place by a silicone cement to avoid bending effects.

The low noise, broad bandwidth of Raman, and low pump power requirements of EDFAs can be combined into one hybrid amplifier to solve amplification issues in long-haul and ultra-long-haul networks. Raman amplifiers provide a gain across a large bandwidth. In contrast, EDFAs provide a substantial gain, but across a relatively small band, and the gain provided is not flat. By combining both, the spectrum achieved is much better than individual configuration.

As shown in Figure 4, a multiwavelength fiber laser based on a hybrid gain medium of Raman-EDF with $17 \mathrm{~m} \mathrm{EDF}, 11 \mathrm{~km}$ SMF, 10:90 pump coupler and 300, 1000 and $1500 \mathrm{~mW}$ pump power have been introduced to overcome this problem. The output power of Hybrid Raman-EDF gain is higher when 1500 $\mathrm{mW}$ pump power was used compared $300 \mathrm{~mW}$ and $1000 \mathrm{~mW}$. This shows the combination between these two media produces more broader gain, so they are able to accommodate more channels. Also, due to Raman amplifiers' non dependence on carrier lifetimes of meta stable states, they can be used at higher bit rates and stable at room temperature.

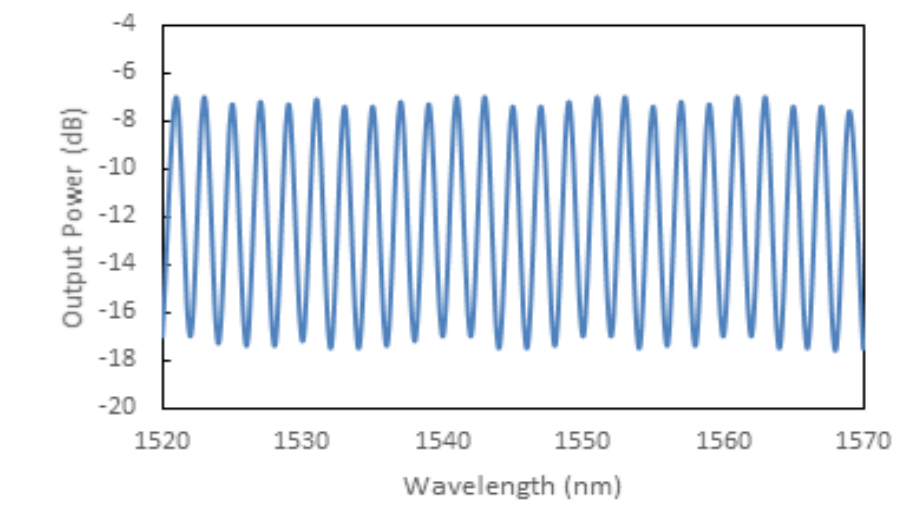

Figure 3. Transmitted laser power versus wavelength for the tapered fiber

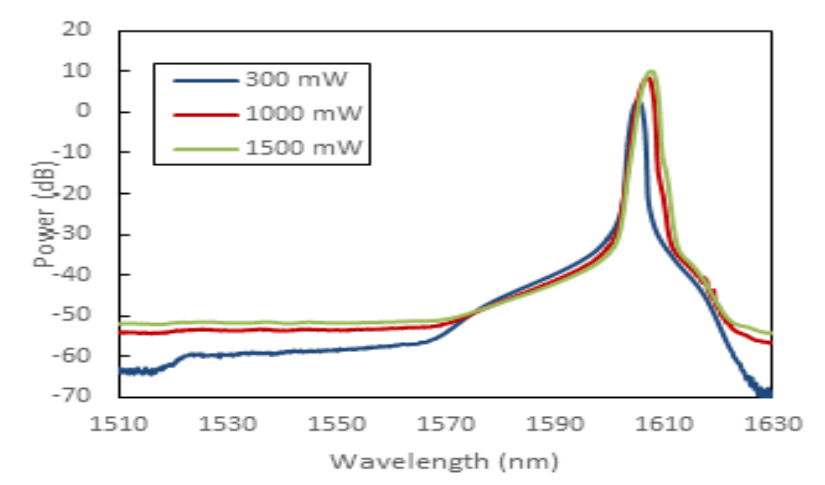

Figure 4. Fiber laser based on a hybrid gain medium of Raman-EDF with different pump power; $300 \mathrm{~mW}$ (blue), $1000 \mathrm{~mW}$ (red) and $1500 \mathrm{~mW}$ (green)

In order to study the multiwavelength effect on the laser, a higher Raman pump power is needed. The hybrid Raman-EDF gain is pumped from the external by RPU. Thus, in this work, a hybrid gain of Raman-EDF is used to boost the laser output power. As a result, the combination of both gains produces a stable multiwavelength with SMSR of $28.9 \mathrm{dBm}$ for $300 \mathrm{~mW}$ pump power, $30.7 \mathrm{dBm}$ for $1000 \mathrm{~mW}$ pump power and $33.7 \mathrm{dBm}$ for $1500 \mathrm{~mW}$ pump power. The generated backward propagating oscillates inside the laser cavity generate the stable multiwavelength output with 4 lasing line for $300 \mathrm{~mW}$ pump power and 2 lasing line for $1000 \mathrm{~mW}$ and $1500 \mathrm{~mW}$ respectively. The output was coupled out via the 10:90 coupler. As shown in Table 1, by varying the pump power used, the number of lasing line, spectral spacing, SMSR 
and peak power also differs. This parameter is defined as the efficiency of the fiber laser to convert pump energy into lasing signal energy.

Table 1. Multiwavelength Laser Specification with 10:90 Coupling Ratio

\begin{tabular}{cccccc}
\hline $\begin{array}{c}\text { Pump } \\
\text { Power }(\mathrm{mW})\end{array}$ & Lasing line & $\begin{array}{c}\text { Spectral Spacing } \\
(\mathrm{nm})\end{array}$ & $\begin{array}{c}\text { Line Width } \\
(\mathrm{nm})\end{array}$ & $\begin{array}{c}\text { SMSR } \\
(\mathrm{dB})\end{array}$ & $\begin{array}{c}\text { Peak Power } \\
(\mathrm{dBm})\end{array}$ \\
\hline 300 & 4 & 3.7 & 0.3 & 28.9 & -6.7 \\
1000 & 2 & 3.9 & 0.3 & 30.7 & 4 \\
1500 & 2 & 4.0 & 0.3 & 33.7 & 5.8 \\
\hline
\end{tabular}

In Figure 5, the multiwavelength laser output spectrum is demonstrated by using different coupler ratios in the configuration of Figure 1 with the pump power between 0.2-1.8 W. If the total hybrid RamanEDF gain is equal to or higher than the cavity loss, multiwavelength laser oscillation can be formed between the optical circulators and $99 \%$ reflective mirror. As shown in Figure 1, the multiwavelength laser is generated with 10:90, coupler, which contributes to higher injected power into the SMF and EDF with lower cavity loss. However, the laser cannot be generated with high efficiency when another type of coupler is applied coupler due to the injected pump power into the SMF which is lower than the threshold and the high loss in the cavity. The highest peak is obtained with a 10:90 coupler which has the lowest cavity loss. The $300 \mathrm{~mW}$ pump power produces a best result for multiwavelength generation. According to results obtained in Figure 5, the 4 simultaneous lines are obtained at approximately $1594.65 \mathrm{~nm}, 1598.1 \mathrm{~nm}, 1602.6 \mathrm{~nm}$, and $1606.5 \mathrm{~nm}$. The $1000 \mathrm{~mW}$ and $1500 \mathrm{~mW}$ power are injected to the cavity and produce 2 channels multiwavelength laser at approximately $1602.6 \mathrm{~nm}$, and $1606.5 \mathrm{~nm}$. The value of SMSR is increased when more power injected through the pump. This happens due to the signal propagates in the opposite direction of the cavity and travels to the circulator, acting here as a reflector, before it is rerouted back into the Raman-EDF gains in the mirror. The amplified signal, then travels back to the other side of reflector and this oscillation continues to generate the higher SMSR and also increases the output power of the laser as shown.

Laser stability is one of the important elements for a laser source. It refers to the temporal stability of the peak power and wavelength of the laser source in the condition that variation in the peak power and the wavelength of the laser source is minimized. Many factors influence a source's stability, such as temperature, pump condition and the gain dynamics. Finally, the output stability testing of proposed single pump multiwavelength fiber laser utilizing tapered EDF based MZI in hybrid Raman-EDF gains design with a linear cavity is made to execute the output performance of power and wavelength. In this experiment, we select one of the lasing lightwave at $1606.05 \mathrm{~nm}$ initially for measurement. Here, the output power was observed every 5 minutes for 2 hours using an optical power meter. As shown in Figure 6 (a) and (b), the peak power and wavelength variations are measured at $\pm 1.8 \mathrm{~dB}, \pm 0.3 \mathrm{~dB}, \pm 0.9 \mathrm{~dB}$ and $\pm 0.2 \mathrm{~nm}, \pm 0.15$ $\mathrm{nm}, \pm 0.1 \mathrm{~nm}$ for $300 \mathrm{~mW}, 1000 \mathrm{~mW}, 1500 \mathrm{~mW}$ pump power, respectively. Hence, the results present that the proposed multiwavelength laser has the excellent optical output stabilities for future system application. In addition, under 2 hours observing time, the measured output stabilities of the proposed laser are still maintained.

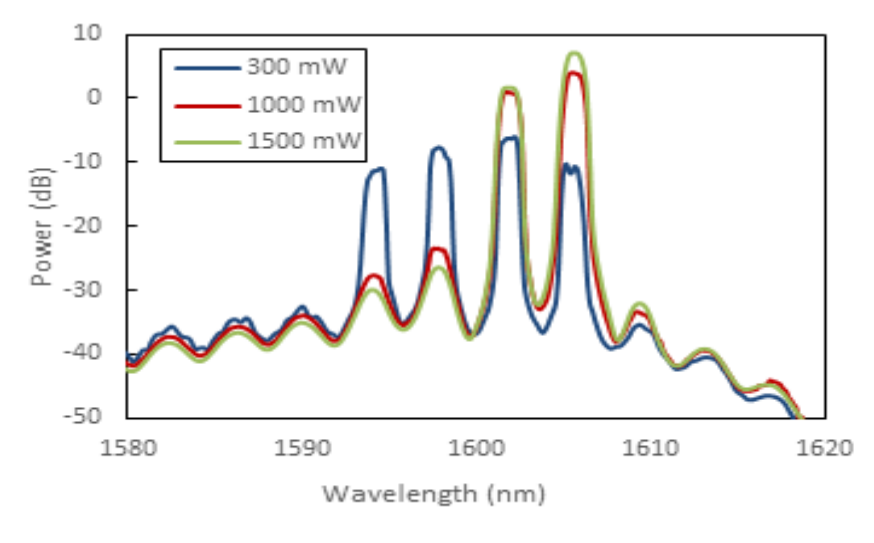

Figure 5. Multiwavelength output signal from single pump laser utilizing Tapered based MZI with 10:90 arm of an optical coupler (OC) 


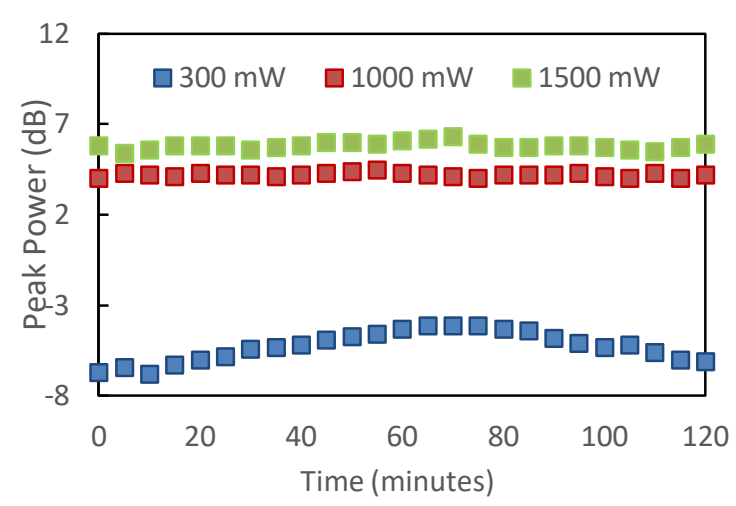

(a)

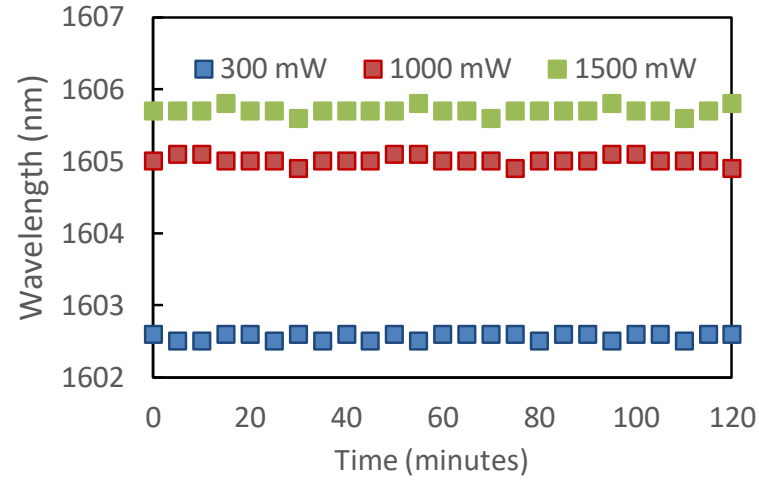

(b)

Figure 6. Stability test of the laser with various pump power (a) Wavelength versus time and (b) Peak power versus time

An important characteristic of a pumped laser source is its slope efficiency. It is obtained by plotting the laser output power against the pump power. Above the threshold power, the curve increases linearly. The efficiency of the laser is given by the slope of this line. This characteristic is due to the same optical loss for all input powers in a cavity. However, the curve may be nonlinear especially for high power lasers, typically with lower slope at high input powers. For an optical laser source, efficiency represents the ratio of the lasing output power over the pump power, in other words, the conversion of the pump energy to the lasing energy. For example, efficiency of a 10:90 coupling ratio laser system has been reported to be about $80 \%$. This value can be obtained from the slope of the transfer characteristic curve as shown in Figure 7.

The minimum amount of pump power required for a laser source to begin lasing is defined as the threshold power. When the pump power is below the threshold power, the output power of the laser source is incoherent and spontaneous. As shown in Figure 7, the threshold power increases as the coupler value increases from $10 \%$ to $90 \%$. The total pump power and output power behavior is initially linear but saturates at high input signal powers. The result shows power conversion efficiency (PCE) has a maximum value when 10:90 coupler was applied.

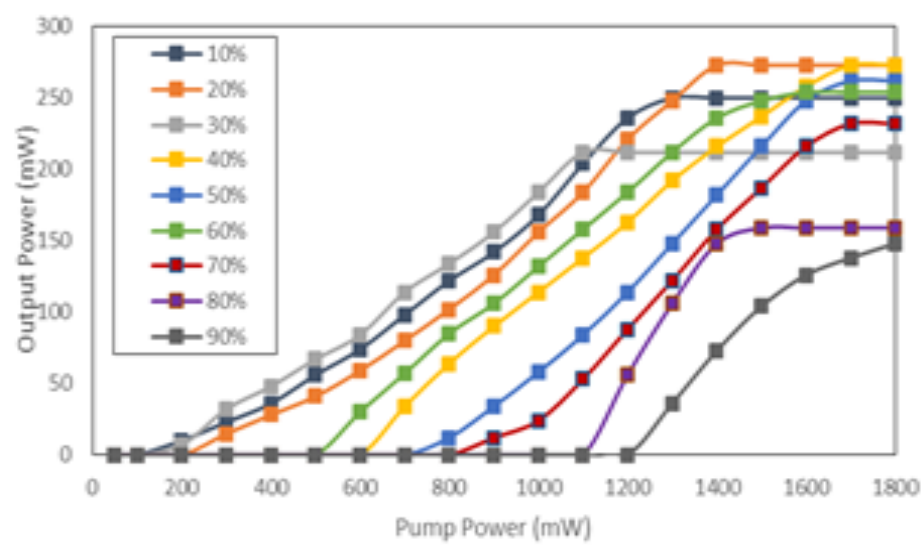

Figure 7. Threshold power graph with different coupling ratio

The interaction between hybrids Raman-EDF is also investigated in the last part of this paper. The results show that the reduction in the threshold power occurs under different Raman pumping power in a linear cavity with $11 \mathrm{~km}$ SMF and $17 \mathrm{~m} \mathrm{EDF}$. The threshold decreases with the increase of Raman-gain OnOff and this decrease is much faster forward pumping than for backward pumping due to the lower Raman gain at this scheme. It is revealed that the amount of threshold reduction depends strongly and solely on the gain effect on it, dependent of Raman pumping power injected to the laser cavity. 
By implementing the reflective mirror at one end, it allows the lasing wavelength to pass the Raman-EDF gain twice per one oscillation and thus increases the net gain per oscillation. This results in the linear cavity to exhibit a lower threshold power and achieves larger SMSR. The proposed cavity using a hybrid Raman-EDF gain medium will allow for the development of compact multiwavelength devices.

\section{CONCLUSION}

This paper presents a multiwavelength laser utilizing a non-adiabatic tapered based-EDF using hybrid Raman and EDF gains in a linear cavity configuration. This tapered-based interferometer has the advantage of simplicity of the fabrications and provides low loss interferometer as compared to its counterpart. A stable output laser of 4 lines is obtained at $300 \mathrm{~mW}$ with SMSR of $28.9 \mathrm{~dB}$ and 2 lines was produced at 1000 and $1500 \mathrm{~mW}$ with SMSR of 30.7 and $33.7 \mathrm{~dB}$ respectively. The laser is very stable at room temperature with peak power variations of 0.3 to $1.8 \mathrm{~dB}$ and wavelength variations of 0.1 to $0.2 \mathrm{~nm}$ for two hours continuous operation.

\section{ACKNOWLEDGEMENTS}

The author would like to acknowledge Faculty of Electrical Engineering, Universiti Teknologi MARA, 40450 Shah Alam, and Kementerian Pendidikan Tinggi Malaysia under grant RAGS/1/2015/TK0/UiTM/03/4 for supporting this project.

\section{REFERENCES}

[1] Mohanad Aljanabi, et al., "Study of impact of art performance level of blue laser technology applications and its control," Indonesian Journal of Electrical Engineering and Computer Science, Vol. 17, No. 3, pp. 1383 1389, March 2020,

[2] Luis C. Básaca-Preciado, et al., "Optical 3D laser measurement system for navigation of autonomous mobile robot," Optics and Lasers in Engineering, Volume 54, Pages 159-169, 2014,

[3] Ionin, A, et al., "Femtosecond laser color marking of metal and semiconductor surfaces," Appl. Phys. A, vol. 107 issue 2 pp 301-305, May 2012.

[4] Y.S. Jeong, et al., "Fiber optic full color synthesizer", Conference on Lasers and Electro-Optics (CLEO), 2005.

[5] P J. Winzer and R-J Essiambre, "Advanced modulation formats for high capacity optical transport networks," J. Lightw. Technol., vol. 24, pp. 4711- 4728, 2006.

[6] C. M. Mclntosh, et al., "Eliminating SRS Channel Depletion in Massive WDM Systems via Optical Filtering Techniques," Photon. Technol. Lett., Vol. 13, pp. 302-304, 2001.

[7] Muhammad Syauqi Kusyairi bin Jamalus, et al., "Simulation of dual stage thulium-doped fiber amplifier using pump power distribution technique," Indonesian Journal of Electrical Engineering and Computer Science, Vol. 15, No. 3, pp. 1203 1211, September 2019.

[8] N A M Ahmad Hambali, et al., "Single-wavelength ring-cavity fiber laser employed preamplification technique to reduce threshold by circulating spontaneous brillouin scattering," Indonesian Journal of Electrical Engineering and Computer Science, Vol. 14, No. 1, pp. 276 283, April 2019.

[9] X. Zhou and M. Brik, "Performance limitation due to statistical Raman crosstalk in a WDM system with multiplewavelength bidirectionally pumped Raman amplification," J. Lightwave Technol., Vol. 21, pp. 2194-2202, 2003.

[10] F. Laser, G. Leader, et al., "Fiber Lasers: Technology, Applications and Associated Laser Safety,” 2012.

[11] Muqing Zhou, et al., "Tunable Multi-Wavelength EDF Laser Based on Sagnac Interferometer with WeaklyCoupled FMF Delay Line," Optical Fiber Communication Conference, OSA Technical Digest paper M2J.5, 2018.

[12] E. Desurvire, et al., "Erbium-Doped Fiber Amplifiers, Device and System Developments," John Wiley, New York, 2002.

[13] L. Jie, et al., "High concentration erbium-doped fiber for wide-band EDFA," Optoelectronics and Communication Conference OECC, pp. 262-263, 2000.

[14] Hariharan, P. "Optical Interferometry” Elsevier/Academic Press: New York, NY, USA, 2003.

[15] [Z. C. Luo, et al., "Tunable and switchable all-fiber comb filter using a PBS-based two-stage cascaded MachZehnder interferometer," Opt. Comm., vol. 284, issue 18, pp. 4167-4170, 2011.

[16] H. Zou, et al., "A wavelength-tunable fiber laser based on a twin-core fiber comb filter," Optics \& Laser Technology, vol. 45, pp. 62933, 2013.

[17] Shan Qin, et al., "Stable and uniform multi-wavelength fiber laser based on hybrid Raman and Erbium-doped fiber gains," Optic Express, vol 14, pp 1113-1118, 2006.

[18] M. D. Mermelstein, et al., "Six-wavelength Raman fibre laser for C- and L-band Raman amplification and dynamic gain flattening," Electron. Lett. Vol. 38, pp. 636-638, 2002.

[19] Shien-Kuei Liaw and Shey-Cheng Liu, "Backward-pumping WDM fiber laser sources using gain fibers of various lengths as self-equalizers “, Technical Digest. CLEO/Pacific Rim 2001. 4th Pacific Rim Conference on Lasers and Electro-Optics, 01TH8557, July 2001. 
[20] Ali, S., et al., "Comparison of the effect structure on Ring and Linear Cavity Lasers of Er-Doped Optical Fibers", International Conference on Computer and Communication Engineering, pp. 546-549, 2008.

[21] G.A.Cárdenas, et al., "Tapered Mach-Zehnder interferometer based on two mechanically induced long-period fiber gratings as refractive index sensor," Optics \& Laser Technology, vol. 10, pp.1016, 2011.

[22] Bing Sun, et al., "Simultaneous measurement of pressure and temperature by employing Fabry-Perot interferometer based on pendant polymer droplet," Opt. Express, vol. 23, pp. 1906-1911, 2015.

[23] Mingran Quan, et al., "Ultra-high sensitivity Fabry-Perot interferometer gas refractive index fiber sensor based on photonic crystal fiber and Vernier effect," Opt. Lett. 40, 4891-4894, 2015.

[24] Li-Yang Shao, et al., "Sensitivity-enhanced temperature sensor with cascaded fiber optic Sagnac interferometers based on Vernier-effect," Optics Communications, vol. 336, Pages 73-76, 2015.

[25] Vanita Bhardwaj and Vinod Kumar Singh, "Fabrication and characterization of cascaded tapered Mach-Zehnder interferometer for refractive index sensing," Sensors and Actuators A: Physical, Vol. 244, 30-34, June 2016.

\section{BIOGRAPHIES OF AUTHORS}

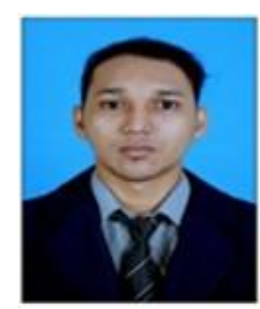

Muhammad Faiz Ibrahim obtained Bachelor of Electronic Engineering (Communication) (Hons) in 2016 from Universiti Teknologi MARA, Shah Alam. He is currently a Researcher at High Voltage Cable Diagnostics, Asset Performance Unit, TNB Research Sdn. Bhd. His research interest is in the area of optical network design, optical communications and network monitoring.

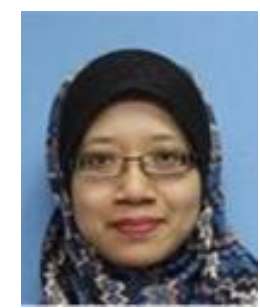

Nani Fadzlina Naim received the B.Eng. degree in Electrical and Electronics Engineering and M.Eng degree in Electronics and Communication Engineering from Universiti Teknologi Malaysia (UTM) in 2005 and 2007, respectively. She received her PhD in 2015 in Electrical, Electronics and Systems Engineering from Universiti Kebangsaan Malaysia (UKM). She is currently a senior lecturer at Faculty of Electrical Engineering, Universiti Teknologi MARA (UiTM). Her current research interests are in the area of design optical networks, optical network monitoring, optical communication, Fiber Bragg Grating sensor, optical sensing technology and its application.

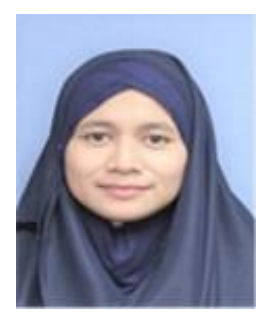

Mas Izyani Md Ali received the B.Eng. (Hons.) in Electrical and Electronics Engineering from University Tenaga Nasional (UNITEN), Selangor, Malaysia, in 2002, and the M.Sc. degree (Research) in Communication and Network Engineering from the University Putra Malaysia in 2007. She graduated with $\mathrm{PhD}$ in 2016 from University Putra Malaysia in Communication and Network Engineering. She is currently a Senior Lecturer at the Faculty of Electrical Engineering, Universiti Teknologi MARA, Shah Alam, Malaysia. Her research interest includes fiber amplifier, fiber laser design, optical communications and optical sensor. 